\title{
Semi-Quantitative Analysis of Brain MR Imaging in 76 Cases of Neonatal Indirect Hyperbilirubinemia
}

\author{
Ruolin Zhang ${ }^{1,2 *}$, Tao Bo ${ }^{1^{*}}$, Li Shen ${ }^{1}$, Senlin Luo ${ }^{1}$, Jian Li ${ }^{1 \#}$ \\ ${ }^{1}$ Department of Pediatrics, Second Xiangya Hospital, Central South University, Changsha, China \\ ${ }^{2}$ Dvision of Neonatology, She Kou People's Hospital, Shenzhen, China \\ Email: "boliy@yeah.net
}

How to cite this paper: Zhang, R.L., Bo, T., Shen, L., Luo, S.L. and Li, J. (2016) SemiQuantitative Analysis of Brain MR Imaging in 76 Cases of Neonatal Indirect Hyperbilirubinemia. Open Journal of Pediatrics, 6, 280-289.

http://dx.doi.org/10.4236/ojped.2016.64039

Received: October 12, 2016

Accepted: November 5, 2016

Published: November 8, 2016

Copyright $\odot 2016$ by authors and Scientific Research Publishing Inc. This work is licensed under the Creative Commons Attribution International License (CC BY 4.0).

http://creativecommons.org/licenses/by/4.0/

\begin{abstract}
Background: Neonatal hyperbilirubinemia is indeed common. However, severe nerve injuries and bilirubin encephalopathy are rare and only occur in the unusual cases of extreme hyperbilirubinemia. Objectives: To investigate brain magnetic resonance imaging (MRI) changes and their correlations with perinatal predisposing factors in neonates with indirect hyperbilirubinemia, via regions of interest (ROIs) analysis. Methods: Seventy-six neonates with a gestational age of $\geq 35$ weeks diagnosed with neonatal indirect hyperbilirubinemia or bilirubin encephalopathy all underwent brain MRI during hospitalization. Depending on peak total serum bilirubin (TSB), they were assigned to group A $(<221 \mu \mathrm{mol} / \mathrm{L}), \mathrm{B}(\geq 221 \mu \mathrm{mol} / \mathrm{L}<342 \mu \mathrm{mol} / \mathrm{L}), \mathrm{C}(\geq 42$ $\mu \mathrm{mol} / \mathrm{L}<428 \mu \mathrm{mol} / \mathrm{L})$, or $\mathrm{D}(\geq 428 \mu \mathrm{mol} / \mathrm{L})$. The globus pallidus and the white matter around the anterior horn of the lateral ventricle and posterior horn of the lateral ventricle were selected as the ROIs. Average optical densities (AODs) of the ROIs and the cerebrospinal fluid region were measured. The ratio between the AODs was designated as the relative optical density (ROD), and used to determine relative signal intensity. Results: RODs of the globus pallidus were significantly lower in group $\mathrm{D}$ than in all other groups. TSB and the ratio of TSB to serum albumin concentration (B/A) was significantly negatively correlated with ROD in theglobus pallidus. Corrected age was significantly negatively correlated with the ROD of the ROIs. Conclusions: Semi-quantitative image analysis is a feasible method to assess conventional brain MRI for neonatal indirect hyperbilirubinemia. The signal intensity of the globus pallidus in T1-weighted images was significantly correlated with TSB level and $\mathrm{B} / \mathrm{A}$.
\end{abstract}

\section{Keywords}

Neonatal Indirect Hyperbilirubinemia, Bilirubin Encephalopathy, Magnetic Resonance Imaging

${ }^{\star}$ These authors contributed equally to this work. 


\section{Introduction}

As one of the most common clinical conditions occurring during the neonatal period, neonatal indirect hyperbilirubinemia has attracted substantial attention as it may cause bilirubin encephalopathy, which describes the clinical central nervous system findings caused by bilirubin toxicity to the basal ganglia and various brainstem nuclei, a kind of severe neonatal brain injuries. The bilirubin encephalopathy are often followed with hemolytic disorder, G6PD deficiency, neonatal sepsis, Crigler-Najjar syndrome and some other incredible factors. With increased scientific understanding of bilirubin encephalopathy, the clinical significance of conventional brain magnetic resonance imaging (MRI) for the diagnosis of bilirubin encephalopathy has also increased [1]. Previous studies found that bilateral, symmetrically-increased T1-weighted image (T1WI) signal in the globus pallidus was a characteristic sign of acute bilirubin encephalopathy, whereas T2-weighted image (T2WI) changes were not obvious [2] [3]. However, there have also been reports that similar changes might also be found in conventional brain MRI of normal neonates [4], raising doubt as to whether these MRI changes are specific to bilirubin encephalopathy. It is well known that the interpretation of conventional brain MR (magnetic resonance) images mainly relies on experienced radiologists, but such interpretation is highly subjective, and inherently prone to bias due to a lack of quantitative or semi-quantitative modes of analysis. The problems associated with fully utilizing conventional brain MRI which hinder accurate analysis the brain damage induced by unconjugated bilirubin (UCB) are in need of immediate solutions.

The aim of this study was to investigate the feasibility of assessing conventional brain MRI via a semi-quantitative analysis of MRI signal intensities from different brain regions in neonatal infants with a gestational age (GA) of $\geq 35$ weeks with indirect hyperbilirubinemia. We hoped to identify characteristic changes in conventional brain MRI and to provide a clear clinical basis to select the proper treatment for early intervention in neonatal infants with hyperbilirubinemia.

\section{Materials and Methods}

\subsection{Subject Selection}

In this study, 1536 patients who were initially diagnosed with neonatal indirect hyperbilirubinemia or bilirubin encephalopathy and admitted to the Second XiangYa Hospital of Central South University between October 2009 and October 2013 were screened according to the following inclusion criteria: 1) The patient was initially diagnosed with neonatal indirect hyperbilirubinemia or bilirubin encephalopathy; 2) complete and thorough clinical data and laboratory data were available; 3) informed consent for MRI was given by the parents of the patients and a conventional MRI scan was performed during hospitalization(average gestational age was $40.6 \pm 1.7$ weeks); 4) the patient was of a GA $\geq 35$ weeks. Patients were excluded if any of the following criteria was met: 1) The patient had brain injuries not induced by bilirubin encephalopathy or congenital brain anomalies; 2) MR images were unclear or had severe artifacts. In all of the 
patients, we excluded 328 patients for uncompleted clinical date and laboratory date. 792 patients refused to conducted brain MR imaging or unnecessary to preform. Beside, 367 patients with the gestational age $<35$ weeks, as well as 37 patients diagnosed with other brain injury and congenial brain anomalies were excluded. Finally eliminated 16 unclear or severely artifact patients, 76 eligible subjects were collected (average gestational age was $38.6 \pm 1.5$ weeks), 43 males and 33 females. The subjects were assigned to groups A, B, C, and D according to their peak total serum bilirubin (TSB) levels (Table 1). Nineteen subjects (25\%) were diagnosed with bilirubin encephalopathy based on clinical symptoms. Whether it with seizure or respiratory failure, there is 12 in the mild bilirubin encephalopathy and 7 in the severe bilirubin encephalopathy. All subjects received phototherapy, however if the TSB level is $25 \mathrm{mg} / \mathrm{dl}$ or higher at any time the infant will conduct exchange transfusion the other proper treatment including pharmacologic treatment and so on. After recovery or alleviation of symptoms, all subjects were underwent conventional brain MRI, and hearing screening at the corrected age (CA) of $\geq 37$ weeks and then discharged.

\subsection{MRI Acquisition and Analysis}

Conventional brain MRI was performed at the MRI Center of the Second XiangYa Hospital of Central South University on a SIEMENS 1.5 T Magnetom Trio Tim System using transverse T1WI and T2WI sequences with a slice thickness of $4 \mathrm{~mm}$, slice gap of $2 \mathrm{~mm}$, matrix of $256 \times 256$, and field of view of $19 \times 19 \mathrm{~cm}$. The horizontal section through the dorsal thalamus, basal ganglia, and caudate nucleus was selected as the section for marker fixation (Figure 1). The globus pallidus as well as the white matter around the anterior horn of the lateral ventricle and the posterior horn of the lateral ventricle were selected as the regions of interest (ROIs), while the CSF region in the same section was used as a control. The average optical densities (AODs) in the ROIs and cerebrospinal fluid (CSF) regions were measured with Image-Pro Plus Version 6.0 software (Media Cybernetics, Inc.). The ratio of AOD in ROIs to AOD in the CSF region was calculated and designated the relative optical density (ROD) of the ROIs, representing their relative signal intensity.

Table 1. Group Assignment for Included Subjects.

\begin{tabular}{|c|c|c|c|c|c|}
\hline \multirow{2}{*}{ Group } & \multirow{2}{*}{$\mathrm{TSB}(\mu \mathrm{mol} / \mathrm{L})$} & \multirow{2}{*}{$\begin{array}{l}\text { Number of } \\
\text { subjects }\end{array}$} & \multirow{2}{*}{$\begin{array}{c}\text { Percentage } \\
(\%)\end{array}$} & \multicolumn{2}{|c|}{ Bilirubin encephalopathy $(n)$} \\
\hline & & & & Mild BE & Severe BE \\
\hline A & $<221$ & $15(0)$ & 19.8 & $1(0)$ & $0(0)$ \\
\hline B & $\geq 221<342$ & $28(6)$ & 36.8 & $3(1)$ & $0(0)$ \\
\hline $\mathrm{C}$ & $\geq 342<428$ & $18(3)$ & 23.7 & $3(0)$ & $3(0)$ \\
\hline \multirow[t]{2}{*}{$\mathrm{D}$} & $\geq 428$ & $15(1)$ & 19.7 & $5(1)$ & $4(0)$ \\
\hline & Total & $76(10)$ & 100.0 & $12(2)$ & $7(0)$ \\
\hline
\end{tabular}

Note: The numbers in parentheses refer to the number of subjects with a GA of $\geq 35$ weeks but $<37$ weeks. TSB, total serum bilirubin. BE, bilirubin encephalopathy. 


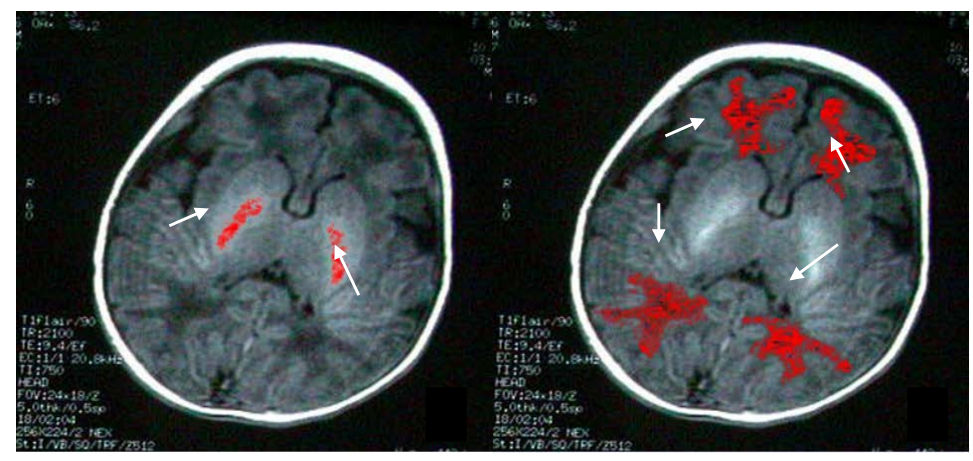

(a)

(b)

Figure 1. ROIs in a Selected Brain MRI (T1WI) Section. (a): Measured globus pallidus region in the selected section (the arrows indicate the selected measured region); (b): Measured the anterior horn of the lateral ventricle; PHLV, posterior horn of the lateral ventricle regions in the selected section (the arrows indicate the selected measured regions). The red enhanced signals in the figure are simply designations of the regions of interest.

The K-S test was used to assess normal distribution of continuous data. The continuous variables with normal distribution are expressed as mean \pm standard deviation. Categorical data are presented as frequencies. Statistical analysis of the results between groups was performed by unpaired Student's t-test or one-way ANOVA. The difference between the frequences of each group were using chi-square test. Data analyses were performed with SPSS 20.0 , and $p<0.05$ was considered statistically significant.

\section{Results}

\subsection{Analysis of ROD Values for ROIs in Conventional Brain MRI}

On the T1WIs of conventional brain MRI, the RODs in the globus pallidus were significantly lower in group D than those in groups A, B, and C; the differences were statistically significant $(p<0.001)$. While the differences in RODs in the globus pallidus between groups $\mathrm{A}, \mathrm{B}$, and $\mathrm{C}$ were not statistically significant $(p>0.05)$, ROD in the globus pallidus gradually decreased as TSB increased (Table 2 ). No statistically significant differences were found in the RODs in the white matter around the anterior horn of the lateral ventricle and the posterior horn of the lateral ventricle among groups $A, B, C$, and D (Table 2). Also, no statistically significant differences were found in the RODs in the white matter around the anterior horn of the lateral ventricle and the posterior horn of the lateral ventricle among the infants with mild bilirubin encephalopathy, the infants with severe bilirubin encephalopathy, and the infants without bilirubin encephalopathy $(p>0.05)$.

T2WI images of conventional brain MRI were also analyzed in this study and no differences in the AOD of the CSF, which was used as control, were found among all subjects in this study, hence this analysis could not be used to objectively calculate the RODs of ROIs. The resu) $1 \mathrm{t}$ of this are main restricted to the Image-Pro Plus 6.0 Media 
Table 2. Comparison of ROD Values of ROIs in Brain MRI T1WIs (Mean \pm SD).

\begin{tabular}{cccc}
\hline Group (n) & ROD in GP & ROD in AHLV & ROD in PHLV \\
\hline A (15) & $0.400 \pm 0.036^{*}$ & $0.733 \pm 0.132$ & $0.769 \pm 0.146$ \\
B (28) & $0.353 \pm 0.077^{*}$ & $0.709 \pm 0.158$ & $0.748 \pm 0.158$ \\
C (18) & $0.369 \pm 0.084^{*}$ & $0.770 \pm 0.100$ & $0.813 \pm 0.091$ \\
D (15) & $0.254 \pm 0.039$ & $0.685 \pm 0.099$ & $0.756 \pm 0.091$ \\
F & 13.926 & 1.398 & 1.218 \\
p & $<0.001$ & 0.251 & 0.310
\end{tabular}

${ }^{\star}$ The differences in pairwise comparisons with Group D were statistically significant $(\mathrm{p}<0.001)$. ROD, relative optical density; ROIs; regions of interest; MRI; magnetic resonance imaging; T1WIs; T1-weighted images; GP, globus pallidus; AHLV, anterior horn of the lateral ventricle; PHLV, posterior horn of the lateral ventricle.

(Cybernetics, Inc). When there is a extremely high optical density, it will difficult to identity.

\subsection{Correlations between Perinatal Risk Factors for Neonatal Hyperbilirubinemia and RODs in ROIs in Brain MRI T1WIs}

With regard to T1WI of brain MRI, the ROD in the globus pallidus was statistically significantly negatively correlated with TSB and the ratio of TSB to serum albumin concentration $(\mathrm{B} / \mathrm{A})(p<0.001)$, whereas there were no statistically significant correlations with other perinatal risk factors $(p>0.05)$ (Table 3$)$. The ROD in the white matter around the anterior horn of the lateral ventricle was significantly negatively correlated with CA and threatened miscarriage $(p<0.05)$, but was not significantly correlated with other perinatal risk factors $(p>0.05)$ (Table 3$)$. CA was significantly negatively correlated with the ROD in the white matter around the PHLV $(p<0.05)$, but there were no statistically significant correlations between this ROD and other perinatal risk factors $(p>0.05)$ (Table 3 ), such as sex, birth weight and other high risk factors.

\subsection{Analysis of Correlations between TSB and RODs in ROIs of Brain MRI T1WIs with Hearing Screening}

Quartile analyses were performed on RODs in ROIs of T1WIs of brain MRI to determine the $25^{\text {th }}$ percentile (P 25) and the $75^{\text {th }}$ percentile (P75) of RODs. The subjects were then divided into three groups according to the RODs in the ROIs, those in the lower quartile $(\leq \mathrm{P} 25)$, those between the upper and lower quartiles $(>\mathrm{P} 25<\mathrm{P} 75)$, and those in the upper quartile ( $\geq$ P75). No significant differences were found between the hearing screening test pass rates of these three groups $(p>0.05)$.

\section{Discussion}

In theory, bilirubin encephalopathy is one of the avoidable diseases among all the diseases that can induce neonatal brain injuries. Effective control over early-stage neonatal indirect hyperbilirubinemia and timely detection of brain injuries for early intervention will improve the prognosis [5] [6]. MRI has gained increasing attention as an important 
tool that assists doctors in the diagnosis of bilirubin encephalopathy. However, there is a need for a more effective method of analyzing MRI changes more accurately and objectively, to facilitate the detection of brain injuries in a timely manner, and provide evidence for intervention [7].

Table 3. Analysis of Correlations Between Perinatal Risk Factors and RODs of ROIs in Brain MRI T1WIs.

\begin{tabular}{|c|c|c|c|c|c|c|c|}
\hline & \multirow[t]{2}{*}{$\begin{array}{l}\text { Count of } \\
\text { category }\end{array}$} & \multicolumn{2}{|c|}{ RODs in GP } & \multicolumn{2}{|c|}{$\begin{array}{l}\text { RODs in white matter } \\
\text { around AHLV }\end{array}$} & \multicolumn{2}{|c|}{$\begin{array}{l}\text { RODs in white matter } \\
\text { around PHLV }\end{array}$} \\
\hline & & $\mathrm{r}$ & $\mathrm{p}$ & $\mathrm{r}$ & $\mathrm{p}$ & $\mathrm{r}$ & $\mathrm{p}$ \\
\hline TSB & & -0.536 & $<0.001^{\star}$ & -0.089 & 0.442 & 0.040 & 0.728 \\
\hline $\mathrm{B} / \mathrm{A}$ value & & -0.485 & $<0.001^{\star}$ & 0.047 & 0.730 & 0.135 & 0.321 \\
\hline $\mathrm{pH}$ value of arterial blood & & 0.043 & 0.719 & 0.020 & 0.869 & -0.023 & 0.848 \\
\hline Sex & & -0.112 & 0.336 & 0.068 & 0.562 & 0.178 & 0.123 \\
\hline CA & & 0.023 & 0.864 & -0.266 & $0.020^{\wedge}$ & -0.242 & $0.036^{\mathbf{\Lambda}}$ \\
\hline Birth weight & & 0.038 & 0.745 & 0.081 & $0.049^{\wedge}$ & 0.114 & 0.330 \\
\hline Age of jaundice onset & & 0.100 & 0.402 & 0.070 & 0.554 & 0.064 & 0.588 \\
\hline 1-minute Apgar score & & 0.144 & 0.432 & 0.136 & 0.458 & 0.033 & 0.857 \\
\hline $\begin{array}{l}\text { Amniotic fluid } \\
\text { contamination }\end{array}$ & 4 & -0.058 & 0.620 & -0.183 & 0.113 & -0.114 & 0.329 \\
\hline $\begin{array}{c}\text { Neonatal } \\
\text { infection/sepsis }\end{array}$ & 65 & 0.078 & 0.502 & 0.133 & 0.254 & 0.125 & 0.282 \\
\hline $\begin{array}{l}\text { Hemolytic disease due to } \\
\text { blood type incompatibility }\end{array}$ & 7 & -0.159 & 0.171 & 0.075 & 0.521 & 0.069 & 0.555 \\
\hline Neonatal anemia & 27 & 0.006 & 0.959 & -0.033 & 0.774 & -0.087 & 0.455 \\
\hline Multiple gestation & & 0.052 & 0.655 & -0.021 & 0.856 & -0.033 & 0.780 \\
\hline G-6-PD deficiency & 2 & 0.176 & 0.128 & 0.128 & 0.272 & 0.143 & 0.219 \\
\hline Gestational infections & 5 & 0.177 & 0.125 & -0.057 & 0.628 & -0.093 & 0.426 \\
\hline Gestational hypertension & 4 & 0.035 & 0.764 & 0.081 & 0.485 & 0.107 & 0.356 \\
\hline $\begin{array}{l}\text { Gestational diabetes } \\
\text { mellitus/abnormal } \\
\text { glucose tolerance }\end{array}$ & 6 & 0.163 & 0.160 & 0.103 & 0.378 & 0.068 & 0.558 \\
\hline $\begin{array}{l}\text { Intrahepatic cholestasis of } \\
\text { pregnancy }\end{array}$ & 2 & 0.010 & 0.934 & 0.059 & 0.613 & 0.023 & 0.845 \\
\hline Threatened miscarriage & 2 & 0.131 & 0.260 & -0.228 & $0.047^{\mathbf{A}}$ & -0.216 & 0.060 \\
\hline $\begin{array}{c}\text { Premature rupture of } \\
\text { membranes }\end{array}$ & 4 & 0.104 & 0.371 & 0.109 & 0.348 & 0.112 & 0.335 \\
\hline Delivery methods & & 0.165 & 0.154 & 0.216 & 0.061 & 0.091 & 0.434 \\
\hline
\end{tabular}

${ }^{*} p<0.05$, indicating statistically significant difference for risk factors; ${ }^{\boldsymbol{\Lambda}} p<0.05$ for risk factors; RODs, relative optical densities; ROIs; regions of interest; MRI; magnetic resonance imaging; T1WIs; T1-weighted images; GP, globus pallidus; AHLV, anterior horn of the lateral ventricle; $\mathrm{PHLV}$, posterior horn of the lateral ventricle; $\mathrm{CA}$, corrected age; TSB, total serum bilirubin; B/A, bilirubin (mg/dL)/albumin (g/L); G-6-PD, glucose-6-phosphate dehydrogenase. 
According to pathological analyses, the regions susceptible to brain injuries induced by indirect hyperbilirubinemia are mainly located in the globus pallidus, and these regions have also exhibited the most significant brain MRI changes in neonatal infants with bilirubin encephalopathy [8] [9]. In this present study, the ROD in the globus pallidus in brain MRI T1WIs was significantly negatively correlated with TSB and B/A, the result support that globus pallidus was one of the sensitive zone in bilirubin encephalopathy. On the other hand, this study reminder us that the change of brain MR imaging emerge more earlier than clinical symptom, even when there is a moderate TSB level. It also implicate that the clinical symptom don't in parallel with the change of brain MR imaging. We can get the conclusion brain MR imaging may be a sensitive index of bilirubin encephalopathy than TSB level or clinical symptom, but the mechanism is still unknown (Table 3). This fact suggests that can the semi-quantitative analysis method presented herein be used to effectively identify damage to the globus pallidus induced by indirect hyperbilirubinemia.

The results of this study revealed that the RODs of the globus pallidus in brain MRI T1WIs were significantly lower in subjects with TSB $\geq 428 \mu \mathrm{mol} / \mathrm{L}$ than those of subjects in other groups. This suggests that globus pallidus damage is more likely to occur when TSB $\geq 428 \mu \mathrm{mol} / \mathrm{L}$. This finding is consistent with the internationally recommended threshold for the use of exchange transfusion, TSB $\geq 428 \mu \mathrm{mol} / \mathrm{L}$ [10]. In addition, this study revealed no significant differences in the RODs of the GP between groups with TSB $<428 \mu \mathrm{mol} / \mathrm{L}$ (groups A, B, and C), but the ROD of the globus pallidus gradually decreased as TSB increased, resulting in a significant negative correlation. This means that the higher the TSB, the more evident the signal changes in the globus pallidus. Previous studies reported that neonatal infants with TSB $<342 \mu \mathrm{mol} / \mathrm{L}$ might also exhibit symptoms of nervous system damage, including cognitive anomalies, movement dysfunction, and hearing impairment, among others [11] [12] [13]; in contrast, some patients with moderate neonatal indirect hyperbilirubinemia might not manifest obvious signs of nervous system anomalies during the neonatal period, but are at significantly increased risk of autism spectrum disorders and attention deficit hyperactivity disorder during early childhood [11] [14] [15] [16] [17]. These findings suggest that although a certain portion of patients with neonatal indirect hyperbilirubinemia did not display typical symptoms of bilirubin encephalopathy, pathological damage to the brain might have already occurred. Further research should be undertaken to investigate the possibility of early detection of potential nervous system damage via globus pallidus signal analysis, so as to achieve early intervention or treatment for improved long-term prognosis.

Some studies have found strong correlations between high UCB levels and axonal injuries, and autopsies have also discovered myelinating disorders and white matter damage in patients with BE [18] [19]. Gkoltsiou et al. [20] reported the MRI manifestation of reduction in white matter volume accompanied by delayed development in infants potentially at risk of damage induced by indirect hyperbilirubinemia during the neonatal period. Therefore, the white matter around the anterior horn of the lateral 
ventricle and the posterior horn of the lateral ventricle were selected as ROIs in this study, but there were no significant differences in the RODs of these regions between groups with different TSB levels, and no significant correlations were found between these RODs and TSB. One possible reason for this is that patients in these groups had acute $\mathrm{BE}$, and dysmyelinating disorders induced by indirect hyperbilirubinemia normally appear after the neonatal period, hence follow-up visits are needed to investigate whether anomalies in the white matter regions are specific to chronic bilirubin encephalopathy. This study also revealed a significantly positive correlation between CA and RODs in the white matter around the anterior horn of the lateral ventricle and the posterior horn of the lateral ventricle. Further research is needed to explore whether this semi-quantitative analysis method can be used to assess the degree of maturity of the white matter around the anterior horn of the lateral ventricle and the posterior horn of the lateral ventricle.

The results of this study revealed no significant difference between the RODs of brain MRI ROIs of neonates at the mild and severe bilirubin encephalopathy and those with indirect hyperbilirubinemia. This might have been because this study was retrospective, and included only a relatively small number of cases of bilirubin encephalopathy. Also, the diagnosis of bilirubin encephalopathy, especially during the Early stage, is based on clinical manifestations, which might cause deviations in results. However, the RODs of the globus pallidus tended to decrease and the RODs of the white matter around the posterior horn of the lateral ventricle gradually increased in severe bilirubin encephalopathy patients, suggesting that during the severe bilirubin encephalopathy not only caused severe damage to the globus pallidus, but might also have impaired the white matter around the cerebral ventricles. Further prospective studies with larger sample sizes are needed to investigate whether this is true.

Conjugation of serum albumin to indirect bilirubin can inhibit the entry of indirect bilirubin through the blood brain barrier, reducing its toxicity to nerve cells, hence the ratio of TSB to serum albumin (bilirubin $[\mathrm{mg} / \mathrm{dL}] /$ albumin $[\mathrm{g} / \mathrm{L}], \mathrm{B} / \mathrm{A}$ ) is an important indicator for predicting the degree of risk for brain injuries caused by UCB [21] [22]. This study revealed that the ROD of the GP in brain MRI T1WIs was significantly negatively correlated with TSB and B/A. This means that an increase in B/A value will lead to an increased possibility of signal anomalies in the globus pallidus, further evidence that $\mathrm{B} / \mathrm{A}$ and $\mathrm{UCB}$ are closely correlated with brain damage.

Due to the limitation of condition, the brainstem auditory evoked response didn't conduct in this study. Therefore, further studies should be conducted to investigate the correlations between brain MRI changes and hearing impairment.

T2WIs of conventional brain MRI were also analyzed in this study, and no differences between the experimental data and the AODs of the CSF, which was used as controls, were found in any subjects in this study; hence, this analysis could not be used to objectively calculate the RODs of ROIs, possibly due to insufficient image resolution and sensitivity of the software used in this study. This problem may be solved by developing semi-quantitative analysis software specific to MRI T2WIs. 


\section{Conclusion}

In conclusion, signal changes in the globus pallidus of conventional brain MRI T1WIs were closely correlated with TSB levels and B/A values. Semi-quantitative image analysis is a feasible method to assess conventional MRI changes in patients with neonatal indirect hyperbilirubinemia.

\section{Fund}

This study was supported by the Science and Technology Plan Program of Hunan Province, No. 14JJ4019.

\section{References}

[1] Hoon Jr., A.J., Stashinko, E.E., Nagae, L.M., et al. (2009) Sensory and Motor Deficits in Children with Cerebral Palsy Born Preterm Correlate with Diffusion Tensor Imaging Abnormalities in Thalamocortical Pathways. Developmental Medicine \& Child Neurology, 51, 697-704. http://dx.doi.org/10.1111/j.1469-8749.2009.03306.x

[2] Sugama, S., Soeda, A. and Eto, Y. (2001) Magnetic Resonance Imaging in Three Children with Kernicterus. Pediatric Neurology, 25, 328-331. http://dx.doi.org/10.1016/S0887-8994(01)00306-X

[3] Wisnowski, J.L., Panigrahy, A., Painter, M.J., et al. (2014) Magnetic Resonance Imaging of Bilirubin Encephalopathy: Current Limitations and Future Promise. Seminars in Perinatology, 38, 422-428. http://dx.doi.org/10.1053/j.semperi.2014.08.005

[4] Barkovich, A.J. (1998) MR of the Normal Neonatal Brain: Assessment of Deep Structures. American Journal of Neuroradiology, 19, 1397-1403.

[5] Maisels, M.J., Watchko, J.F., Bhutani, V.K., et al. (2012) An Approach to the Management of Hyperbilirubinemia in the Preterm Infant Less than 35 Weeks of Gestation. Journal of Perinatology, 32, 660-664. http://dx.doi.org/10.1038/jp.2012.71

[6] Hansen, T.W. (2011) Prevention of Neurodevelopmental Sequelae of Jaundice in the Newborn. Developmental Medicine \& Child Neurology, 53, 24-28. http://dx.doi.org/10.1111/j.1469-8749.2011.04059.x

[7] Rose, J. and Vassar, R. (2015) Movement Disorders due to Bilirubin Toxicity. Seminars in Fetal and Neonatal Medicine, 20, 20-25. http://dx.doi.org/10.1016/j.siny.2014.11.002

[8] Johnston, M.V. and Hoon Jr., A.H. (2000) Possible Mechanisms in Infants for Selective Basal Ganglia Damage from Asphyxia, Kernicterus, or Mitochondrial Encephalopathies. Journal of Child Neurology, 15, 588-591. http://dx.doi.org/10.1177/088307380001500904

[9] Steinborn, M., Seelos, K.C., Heuck, A., et al. (1999) MR Findings in a Patient with Kernicterus. European Radiology, 9, 1913-1915. http://dx.doi.org/10.1007/s003300050947

[10] American Academy of Pediatrics Subcommittee on Hyperbilirubinemia (2004) Management of Hyperbilirubinemia in the Newborn Infant 35 or More Weeks of Gestation. Pediatrics, 114, 297-316. http://dx.doi.org/10.1542/peds.114.1.297

[11] Piek, J.P., Dawson, L., Smith, L.M., et al. (2008) The Role of Early Fine and Gross Motor Development on Later Motor and Cognitive Ability. Human Movement Science, 27, 668681. http://dx.doi.org/10.1016/j.humov.2007.11.002

[12] Von Hofsten, C. (2009) Action, the Foundation for Cognitive Development. Scandinavian Journal of Psychology, 50, 617-623. http://dx.doi.org/10.1111/j.1467-9450.2009.00780.x

[13] Gallese, V., Rochat, M., Cossu, G., et al. (2009) Motor Cognition and Its Role in the Phylo- 
geny and Ontogeny of Action Understanding. Developmental Psychology, 45, 103-113. http://dx.doi.org/10.1037/a0014436

[14] Shapiro, S.M. (2003) Bilirubin Toxicity in the Developing Nervous System. Pediatric Neurology, 29, 410-421. http://dx.doi.org/10.1016/j.pediatrneurol.2003.09.011

[15] Burke, B.L., Robbins, J.M., Bird, T.M., et al. (2009) Trends in Hospitalizations for Neonatal Jaundice and Kernicterus in the United States, 1988-2005. Pediatrics, 123, 524-532. http://dx.doi.org/10.1542/peds.2007-2915

[16] Shapiro, S.M. and Popelka, G.R. (2011) Auditory Impairment in Infants at Risk for Bilirubin-Induced Neurologic Dysfunction. Seminars in Perinatology, 35, 162-170. http://dx.doi.org/10.1053/j.semperi.2011.02.011

[17] Johnson, L. and Bhutani, V.K. (2011) The Clinical Syndrome of Bilirubin-Induced Neurologic Dysfunction. Seminars in Perinatology, 35, 101-113. http://dx.doi.org/10.1053/j.semperi.2011.02.003

[18] Barateiro, A., Vaz, A.R., Silva, S.L., Fernandes, A. and Brites, D. (2012) ER Stress, Mitochondrial Dysfunction and Calpain/JNK Activation Are Involved in Oligodendrocyte Precursor Cell Death by Unconjugated Bilirubin. Neuromolecular Medicine, 14, 285-302. http://dx.doi.org/10.1007/s12017-012-8187-9

[19] Barateiro, A., Miron, V.E., Santos, S.D., Relvas, J.B. and Fernandes, A. (2013) Unconjugated Bilirubin Restricts Oligodendrocyte Differentiation and Axonal Myelination. Molecular Neurobiology, 47, 632-644. http://dx.doi.org/10.1007/s12035-012-8364-8

[20] Gkoltsiou, K., Tzoufi, M., Counsell, S., Rutherford, M. and Cowan, F. (2008) Serial Brain MRI and Ultrasound Findings: Relation to Gestational Age, Bilirubin Level, Neonatal Neurologic Status and Neurodevelopmental Outcome in Infants at Risk of Kernicterus. Early Human Development, 84, 829-838. http://dx.doi.org/10.1016/j.earlhumdev.2008.09.008

[21] Ahlfors, C.E. (1994) Criteria for Exchange Transfusion in Jaundiced Newborns. Pediatrics, 93, 488-494.

[22] Iskander, I., Gamaleldin, R., El Houchi, S., et al. (2014) Serum Bilirubin and Bilirubin/Albumin Ratio as Predictors of Bilirubin Encephalopathy. Pediatrics, 134, e1330-e1339. http://dx.doi.org/10.1542/peds.2013-1764

Submit or recommend next manuscript to SCIRP and we will provide best service for you:

Accepting pre-submission inquiries through Email, Facebook, LinkedIn, Twitter, etc. A wide selection of journals (inclusive of 9 subjects, more than 200 journals)

Providing 24-hour high-quality service

User-friendly online submission system

Fair and swift peer-review system

Efficient typesetting and proofreading procedure

Display of the result of downloads and visits, as well as the number of cited articles Maximum dissemination of your research work

Submit your manuscript at: http://papersubmission.scirp.org/

Or contact ojped@scirp.org 